\title{
QUEEN'S
UNIVERSITY
BELFAST
}

\section{Optical Nonlocalities and Additional Waves in Epsilon-Near-Zero Metamaterials}

Pollard, R., Murphy, A., Hendren, W., Evans, P., Atkinson, R., Wurtz, G., Zayats, A., \& Podolskiy, V. A. (2009). Optical Nonlocalities and Additional Waves in Epsilon-Near-Zero Metamaterials. Physical Review Letters, 102(12), [127405]. https://doi.org/10.1103/PhysRevLett.102.127405

\section{Published in:}

Physical Review Letters

Document Version:

Publisher's PDF, also known as Version of record

Queen's University Belfast - Research Portal:

Link to publication record in Queen's University Belfast Research Portal

Publisher rights

(C) 2009 The American Physical Society

\section{General rights}

Copyright for the publications made accessible via the Queen's University Belfast Research Portal is retained by the author(s) and / or other copyright owners and it is a condition of accessing these publications that users recognise and abide by the legal requirements associated with these rights.

Take down policy

The Research Portal is Queen's institutional repository that provides access to Queen's research output. Every effort has been made to ensure that content in the Research Portal does not infringe any person's rights, or applicable UK laws. If you discover content in the Research Portal that you believe breaches copyright or violates any law, please contact openaccess@qub.ac.uk. 


\title{
Optical Nonlocalities and Additional Waves in Epsilon-Near-Zero Metamaterials
}

\author{
R. J. Pollard, A. Murphy, W. R. Hendren, P. R. Evans, R. Atkinson, G. A. Wurtz, and A. V. Zayats \\ Centre for Nanostructured Media, The Queen's University of Belfast, Belfast, BT7 1NN, United Kingdom
}

Viktor A. Podolskiy*

Physics Department, Oregon State University, 301 Weniger Hall, Corvallis, Oregon 97331, USA

(Received 18 December 2008; revised manuscript received 20 February 2009; published 27 March 2009)

\begin{abstract}
We analyze the optical properties of plasmonic nanorod metamaterials in the $\varepsilon$-near-zero regime and show, both theoretically and experimentally, that the performance of these composites is strongly affected by nonlocal response of the effective permittivity tensor. We provide the evidence of interference between main and additional waves propagating in the room-temperature nanorod metamaterials and develop an analytical description of this phenomenon. Additional waves are present in the majority of low-loss $\varepsilon$-near-zero structures and should be explicitly considered when designing applications of $\varepsilon$-near-zero composites, as they represent a separate communication channel.
\end{abstract}

Metamaterials, optical composites with structural units smaller than the wavelength, promise to provide the platform for realization of previously unachievable applications, such as superlensing [1], optical cloaking [2], photonic circuits $[3,4]$, and multiscale light coupling [5,6]. Many of these applications make use of epsilon-nearzero (ENZ) metamaterials in which components of the real part of dielectric permittivity $\operatorname{Re} \varepsilon$ become vanishingly small. Here we show that in the limit of small losses, the optical properties of room-temperature ENZ metamaterial systems are strongly affected by a nonlocal response of effective dielectric permittivity. This behavior is remarkably similar to the behavior of crystalline solids at ultralow temperatures [7-9].

The electromagnetic response of nanorod metamaterials has been a subject of several recent studies [10-17]. It has been shown that these structures behave as uniaxial metamaterials with the optical axis parallel to rods. In general, the properties of these systems are well described by the Maxwell-Garnett-type effective medium theories (EMTs) $[10,13,15]$. Several studies have identified deviations of electromagnetic behavior of nanorod metamaterials from the effective medium approximation due to spatial dispersion, signified by the shift of dispersion curves from local EMT predictions, most notably at relatively low frequencies [16]. Nonlocality-based corrections to imaging and guiding in low-frequency wire arrays have been analyzed [18].

In this Letter we demonstrate experimentally and theoretically that in the optical ENZ regime the spatial dispersion qualitatively changes the optical properties of structures and leads to excitation of additional transversemagnetic-polarized waves that do not exist in local EMTs. In particular, we demonstrate experimental evidence of excitation of additional waves in anisotropic nanorod-based metamaterials and provide the theoretical description of this phenomenon. Additional waves, which are expected to be present in most low-loss ENZ metamaterials, represent a new information channel in these systems, and therefore may lead to new exciting applications of ENZ composites.

The metamaterials studied in this Letter are formed by assembly of aligned metallic ( $\mathrm{Au}$ ) nanorods embedded in an alumina matrix (Fig. 1). The gold nanorods were grown by direct electrodeposition into the pores formed in thin film anodized aluminum oxide, as described in detail in Ref. [19]. The typical parameters of the nanorod systems studied here are $r=12.5 \mathrm{~nm}$ radius, $300 \mathrm{~nm}$ length with $d=60 \mathrm{~nm}$ separation between the rods in the array ( $r$, $d \ll \lambda$, with $\lambda$ being free-space wavelength). For spectral measurements, the samples were illuminated by collimated light (diameter $5 \mathrm{~mm}$ ) from the tungsten-halogen source, and the transmitted light was collected into the fiber bundle coupled to the spectrograph with the liquid-nitrogencooled CCD array.

The transmission spectrum of nanorod metamaterials at oblique incidence for $p$-polarized incident light is dominated by two well-defined features [Figs. 1(c) and 1(d)] whose physical nature is associated with free-electron excitation perpendicular and along the nanorod axis, respectively, excited by the electric field of the incident light $[10,12]$. These transverse $(T)$ and longitudinal $(L)$ modes correspond to the resonances $\left|\varepsilon_{y z}\right| \gg 1$ and $\varepsilon_{x} \rightarrow 0$, respectively.

The strength and width of the $L$ resonances are essentially limited by the optical absorption in plasmonic nanorods which is responsible for the deviation of $\operatorname{Re} \varepsilon_{x}$ from zero. In contrast to thin-film-based plasmonic metamaterials, the internal structure of solution-derived gold strongly limits the electron motion in the metal. Quantitatively, the effect of the restricted mean free path of the electrons on the permittivity of gold is described by [20]

$$
\varepsilon_{\mathrm{Au}}(\omega)=\varepsilon_{\mathrm{bulk}}+\frac{i \omega_{p}^{2} \tau\left(R_{b}-R\right)}{\omega(\omega \tau+i)\left(\omega \tau R+i R_{b}\right)}, \quad R \leq R_{b},
$$



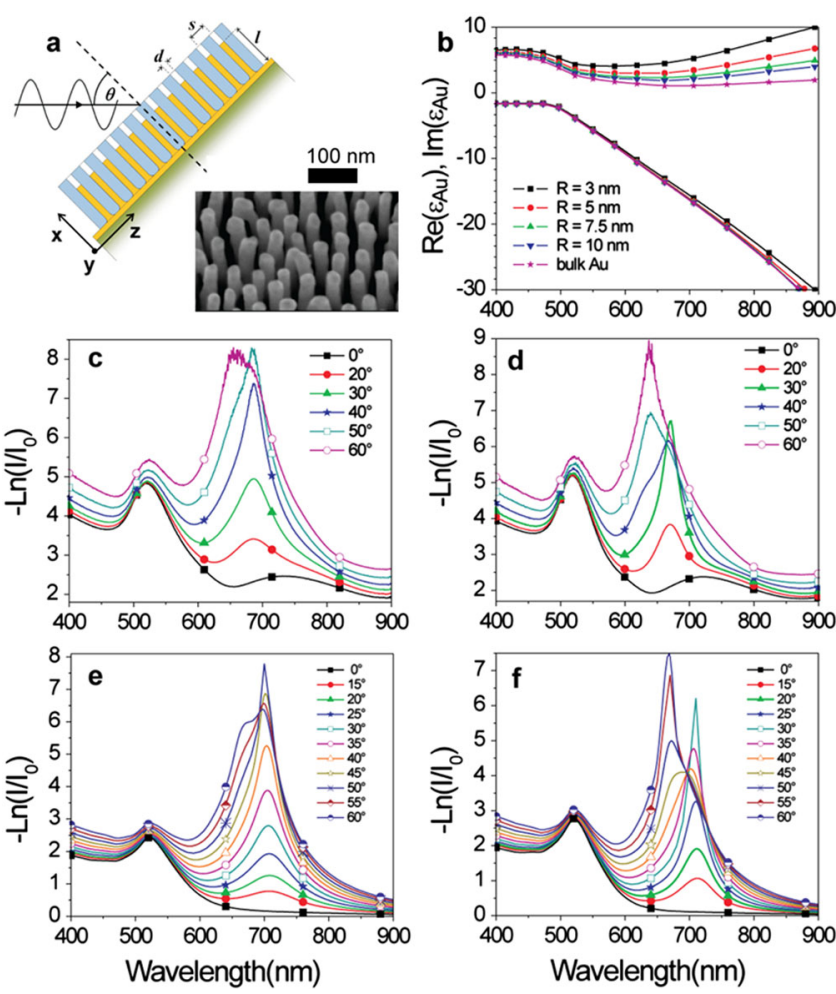

FIG. 1 (color online). (a) Schematic of the metamaterial and the optical measurements and the scanning electron microscopy image of the nanorods after the removal of the anodized aluminium oxide matrix. (b) Spectra of Au permittivity calculated for different values of parameter $R$ in Eq. (1). (c, d) Extinction spectra measured with $p$-polarized incident light for different angles of incidence with the nanorod assemblies unannealed (c) and annealed at $300{ }^{\circ} \mathrm{C}$ for 2 hours (d). (e, f) Extinction spectra derived from 3D FEM-based simulations for the $\varepsilon_{\mathrm{Au}}$ corresponding to $R=5 \mathrm{~nm}$ (e) and $R=10 \mathrm{~nm}$ (f).

where $\varepsilon_{\text {bulk }}$ is the permittivity of bulk metal [21], $R_{b} \simeq$ $35.7 \mathrm{~nm}$ is the mean free path of the electrons in bulk Au, $\omega_{p} \simeq 13.7 \times 10^{15} \mathrm{~Hz}$ is the plasma frequency, $\tau \simeq 2.53 \times$ $10^{-14} \mathrm{~s}$ is the relaxation time for the free electrons in gold, and $R$ is the effective (restricted) mean free path. For solution-based gold nanorods, it is not untypical to have $R \sim 3 \mathrm{~nm}$. Plane-wave-extinction spectra of periodic nanorod metamaterials for different values of parameter $R$ were simulated with a three-dimensional (3D) finiteelement-method (FEM) solver [22]. Results of these simulations, shown in Figs. 1(e) and 1(f), clearly predict the splitting of $L$ resonance in systems with larger $R$ (in all cases $R<r$ ).

The unique advantage of nanorod composites over the typical metamaterial is that the electron confinement scale (and thus the absorption) may be drastically changed by annealing the samples. The internal structure of $\mathrm{Au}$ in nanords changes during annealing so that the electron mean free path can be controlled. In our studies, the samples were annealed in different regimes $\left(100-300^{\circ} \mathrm{C}\right.$ for 1-3 hours), resulting in the estimated increase of the restricted mean free path from $3 \mathrm{~nm}$ to $10 \mathrm{~nm}$. The spec- troscopic measurements reveal that the changes in the permittivity of the metal and, particularly, the decrease in the imaginary part of permittivity, have a very minor effect on $T$ resonance, but lead to splitting of the $L$ resonance in lower-loss systems. In agreement with 3D simulations, the splitting strongly depends on the direction of wave propagation (incident angle) and on absorption (Fig. 1).

To gain insight into the origin of this splitting, we analyzed the dispersion of the eigenmodes of the periodic nanorod composites with FEM-based [22] simulations. The simulations reveal that the system supports three different waves: one transverse-electric wave (responsible for the $T$ resonance) and two transverse-magnetic (TM) waves. The $L$ resonance coincides with a regime of strong interaction between the two TM waves, signified by the avoided crossing between their dispersion curves. This is shown in Fig. 2(b) where the dispersion of the two TM modes obtained from FEM-based solutions of Maxwell's equations is shown with dots. For simplicity, the behavior of the square of the modal index $\operatorname{Re}\left(n^{2}\right)=\operatorname{Re}\left(k_{x}^{2} c^{2} / \omega^{2}\right)$ has been considered. If this quantity is positive, the electromagnetic wave propagates in the medium; if the quantity is negative, the wave exponentially decays into the me-
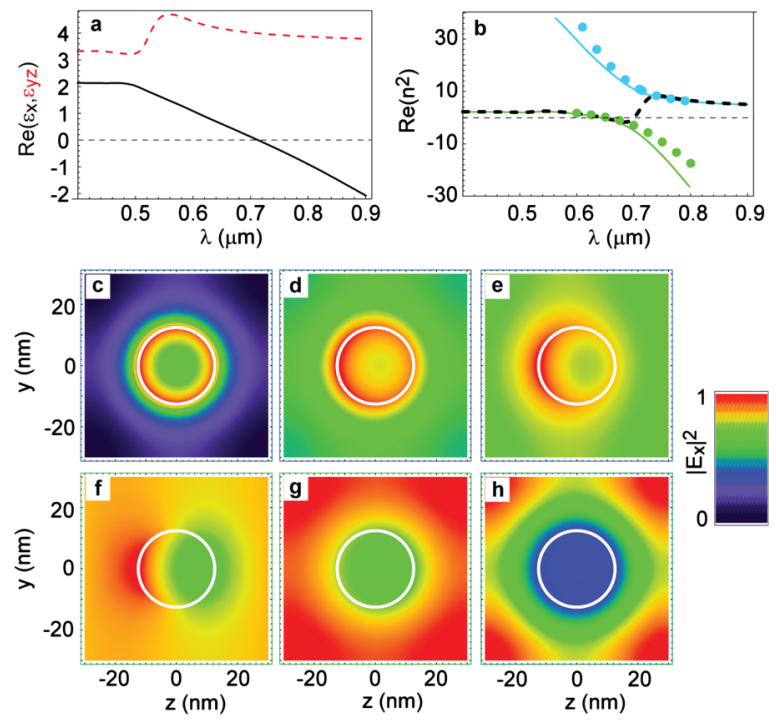

FIG. 2 (color online). (a) Effective permittivities (real part) of nanorod metamaterial calculated with local EMT for $R=$ $10 \mathrm{~nm}$; solid and dashed lines correspond to $\varepsilon_{x}$ and $\varepsilon_{y z}$, respectively; nonlocal corrections are observed near $\varepsilon_{x}=0$. (b) Dispersion of the two TM-polarized modes calculated according to numerical simulations (dots) and after Eq. (5) (solid lines); dashed black line corresponds to the main wave described by the local EMT. (c-h) The profiles of the electric field in the unit cell around the nanorod for the upper (c-e) and lower (f-h) branches for $\theta=60^{\circ}$ : (c, f) $\lambda=600 \mathrm{~nm}$, the "main" mode with uniform field (f) and additional wave with strongly nonuniform field (c) can be clearly distinguished; (d, g) $\lambda=700 \mathrm{~nm}$, regime of strong mode mixing; the profile of both modes is almost uniform; (e, h) $\lambda=800 \mathrm{~nm}$, the main (e) and additional (h) waves can be distinguished again. 
dium. It is clearly seen that there exists a range of wavelengths shorter than the $L$ resonance where both TMpolarized waves simultaneously propagate in the metamaterial. Moreover, this behavior is identical to the response of nonlocal nongyrotropic crystalline materials [7].

In metamaterials, as well as in crystalline media, spatial dispersion (caused by the field variation at the scale of material inhomogeneity) represents a relatively weak correction to effective permittivity:

$$
\varepsilon=\varepsilon^{0}(\omega)+\delta_{\varepsilon}(\vec{k}), \quad\left|\delta_{\varepsilon}\right| \ll 1 .
$$

Thus, the effects caused by the spatial dispersion can be typically neglected [15]. However, in proximity to the ENZ condition, the contribution of spatial dispersion becomes increasingly important. The presence of strong nonlocal terms dramatically changes the optical properties of materials, and often leads to the appearance of the additional transverse or longitudinal waves [8]. In conventional materials, nonlocality-induced additional waves can only be observed at very low temperatures when losses are low $[8,9]$. Metamaterials enable excitation, analysis, and utilization of these waves at room temperatures [23].

The two TM waves identified in our numerical simulations represent main and additional TM waves supported by the nanorod metamaterial. The main wave with relatively smooth $E_{x}$ profile [Figs. 2(e) and 2(f)] corresponds to the lower TM mode for wavelength below the $L$ resonance and to the upper TM mode for larger wavelengths. Note that the behavior of this mode away from $L$ resonance is adequately described by local effective medium theory [dashed line in Fig. 2(b)]. At the same frequency range, the additional wave has strongly nonuniform field distribution [Figs. 2(c) and 2(h)] and can only weakly couple to incident plane waves. The strong interaction between these modes in proximity to $L$ resonance leads to substantial field mixing. As a result of this process, the profiles of both main and additional waves become almost uniform, and both waves contribute to the optical response of the structure.

To quantitatively describe the behavior of $L$ resonance, we follow the procedures introduced in Ref. [7], and represent the permittivity as

$$
\varepsilon_{x}=\varepsilon_{x}^{0}(\omega)-\delta_{x} \frac{k_{x}^{2} c^{2}}{\omega^{2}}, \quad \varepsilon_{y z}=\varepsilon_{y z}^{0}(\omega),
$$

with components of tensor of $\varepsilon^{0}(\omega)$ described by local effective medium theory $[10,13,15]$ :

$$
\begin{aligned}
\varepsilon_{x}^{0} & =p \varepsilon_{\mathrm{Au}}+(1-p) \varepsilon_{\mathrm{Al}_{2} \mathrm{O}_{3},} \\
\varepsilon_{y z}^{0} & =\frac{p \varepsilon_{\mathrm{Au}} E_{\mathrm{Au}}+(1-p) \varepsilon_{\mathrm{Al}_{2} \mathrm{O}_{3}} E_{0}}{p E_{\mathrm{Au}}+(1-p) E_{0}},
\end{aligned}
$$

with $E_{\mathrm{Au}}=2 E_{0} \varepsilon_{\mathrm{Al}_{2} \mathrm{O}_{3}} /\left(\varepsilon_{\mathrm{Au}}+\varepsilon_{\mathrm{Al}_{2} \mathrm{O}_{3}}\right)$, and parameters $p$, $\varepsilon_{\mathrm{Au}}$, and $\varepsilon_{\mathrm{Al}_{2} \mathrm{O}_{3}}$ corresponding to the (surface) concentration of pores in alumina matrix (filling factor), and the permittivity of metamaterial components, respectively.
The dispersion of the three waves of anisotropic metamaterial (propagating in the $x z$ plane) is now given by

$$
\begin{aligned}
k_{x}^{2}+k_{z}^{2} & =\frac{\omega^{2}}{c^{2}} \varepsilon_{y z}^{0}, \\
2 \delta_{x} k_{x}^{2} & =\frac{\omega^{2}}{c^{2}}\left[\varepsilon_{x}^{0}+\delta_{x} \varepsilon_{y z}^{0} \pm \sqrt{\left(\varepsilon_{x}^{0}-\delta_{x} \varepsilon_{y z}^{0}\right)^{2}+4 \varepsilon_{y z}^{0} \delta_{x} \frac{k_{z}^{2} c^{2}}{\omega^{2}}}\right],
\end{aligned}
$$

with $k_{x}$ and $k_{z}[=\omega / c \sin (\theta)]$ being the components of the modal wave vectors.

The value of $\delta_{x}$ can be obtained by fitting Eq. (5) to FEM-based solutions. Such a procedure, performed for a number of values of $k_{z}$ and $R$, results in $\delta_{x} \simeq 0.04-$ $0.006 i$. Note that this value is comparable with the one typically achieved in ultralow-temperature semiconductors [8]. The agreement between the analytical calculation Eq. (5) and the results of FEM simulations is shown in Fig. 2.

The analytical results provide an understanding of the electromagnetic behavior of anisotropic metamaterials in the ENZ regime. For relatively large losses, the contribution of nonlocality of permittivity is weak, and the system is adequately described by the local dielectric function. Although the additional wave does exist, the wavelength range corresponding to the avoided crossing between the two modes is extremely narrow. In the absence of mode mixing (which is restricted to the regime of avoided crossing), the spatial profile of the additional wave prevents it from coupling to the incident plane waves. Therefore, the extinction at the $L$ resonance is dominated by the imaginary part of the wave vector of the main wave. Since in the ENZ regime $\operatorname{Im}\left[k_{x}\right] \sim \omega \sin \theta \operatorname{Re}\left[\sqrt{\varepsilon_{y z} / \varepsilon_{x}}\right] / c \gg \omega / c$, only intensity, but not the spectral location of the $L$ resonance, depends on the incident angle.

The situation changes dramatically when the wave-vector-dependent term in Eq. (3) becomes comparable with the leading $\omega$-dependent term, which can be the case for higher angles [larger $k_{z}$, Fig. 3(a)] or small absorption [Fig. 3(d)]. Here the strong nonlocality results in substantial interaction between the "main" and "additional" TMpolarized waves that is accompanied by substantial field mixing as described above. As a result of this interaction and field mixing, both waves significantly contribute to transmission through the metamaterial.

The simultaneous coupling of both waves to the incident radiation has two main consequences. First, both intensity and spectral location of the extinction resonance, corresponding to the $\operatorname{Im} k_{x}$ maximum of the main wave, depend on the incident angle. Second, the additional wave effectively opens up an additional communication channel in the system. As result, the extinction resonance (of the main wave) is modulated by the interference between main and additional waves.

Note that both TM-polarized modes simultaneously exist in the nonlocal metamaterial. Since these waves have 

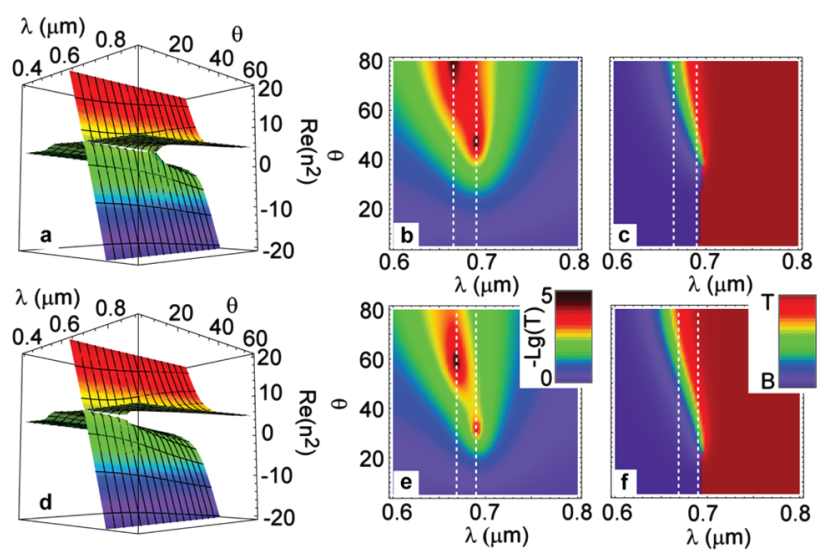

FIG. 3 (color online). (a, d) The dependence of dispersion of two TM-polarized modes on material absorption: $R=5 \mathrm{~nm}$ (a) and $R=10 \mathrm{~nm}(\mathrm{~d})$. The decrease in absorption enhances the mode mixing, signified by the avoided crossing between the dispersion curves. Panels (b) and (e) show the extinction profile of the systems in (a) and (d), respectively. Panels (c) and (f) represent the ratio of amplitudes of the two waves at the exit side (metamaterial-glass interface) of the structures in (a) and (d). " $B$ " and " $T$ " correspond to the bottom and top modes in $(\mathrm{a}, \mathrm{d})$; white lines show the spectral positions of the two extinction maxima.

identical polarization, it is impossible to determine their relative contribution to the field inside the system via conventional boundary conditions. Over the years, several forms of additional boundary conditions have been proposed to resolve this ambiguity in crystalline materials [8]. Our analysis suggests that the condition $\vec{P}_{\text {nonlocal }}=0$ adequately describes the optical response of metamaterials.

Results of transfer-matrix wave-matching simulations incorporating the nonlocal EMT [Eq. (5)] and the above additional boundary condition, shown in Figs. 3(b) and 3(e), are in agreement with the experiments and with 3D FEM simulations. Note that for a range of frequencies and incident angles around $L$ resonances, the amplitudes of both waves at the exit side of the metamaterial are equal to each other [Figs. 3(c) and 3(f)] with the transmission through the structure related to the interference between these two waves. The points of destructive interference correspond to transmission minima or, equivalently, to extinction maxima. While only two such maxima are seen in our experiment, our simulations suggest that higher-order interference maxima will be seen for longer nanorods.

To conclude, we have demonstrated that the optical properties of metamaterials may strongly deviate from the predictions of local effective medium theories in the ENZ regime. Strong nonlocality is accompanied by excitation of the additional (TM-polarized) electromagnetic wave. The propagation of this wave has to be taken into account when predicting the optical properties of metamaterials. Note that the additional wave not only distorts the propagation of its main counterpart, but it also represents an additional information channel in the system, and thus can be beneficial for some applications. The evidence of excitation of an additional wave in nanorod-based metamaterials has been found experimentally. An analytical description of the optical response of nonlocal ENZ nanorods has been presented.

This work has been supported by EPSRC (UK) and by ONR (Grant No. N00014-07-1-0457), NSF (Grant No. ECCS-0724763), and ACS-PRF.

*viktor.podolskiy@physics.oregonstate.edu

[1] J. B. Pendry, Phys. Rev. Lett. 85, 3966 (2000).

[2] L.S. Dolin, Izv. VUZov Radiofizika 4, 964 (1961); J. B. Pendry, D. Schurig, and D. Smith, Science 312, 1780 (2006); W. Cai, U. K. Chettiar, A. V. Kildishev, and V. M. Shalaev, Nat. Photon. 1, 224 (2007).

[3] M. Silveirinha and N. Engheta, Phys. Rev. Lett. 97, 157403 (2006).

[4] N. Engheta, Science 317, 1698 (2007).

[5] Z. Jacob, L. V. Alekseyev, and E. Narimanov, Opt. Express, 14, 8247 (2006); A. Salandrino and N. Engheta, Phys. Rev. B 74, 075103 (2006); Z. Liu et al., Science, 315, 1686 (2007).

[6] R. W. Ziolkowski, Phys. Rev. E 70, 046608 (2004).

[7] S. I. Pekar, Sov. Phys. JETP 6, 785 (1958).

[8] V. M. Agranovich and V.L. Ginzburg, Crystal Optics with Spatial Dispersion and Excitons, Springer Series in SolidState Sciences Vol. 42 (Springer-Verlag, Berlin, 1984).

[9] J. J. Hopfield and D. G. Thomas, Phys. Rev. 132, 563 (1963).

[10] R. Atkinson et al., Phys. Rev. B 73, 235402 (2006).

[11] W. Dickson et al., Phys. Rev. B 76, 115411 (2007).

[12] G. A. Wurtz et al., Opt. Express 16, 7460 (2008).

[13] A. N. Lagarkov and A. K. Sarychev, Phys. Rev. B 53, 6318 (1996).

[14] J. B. Pendry et al., Phys. Rev. Lett. 76, 4773 (1996).

[15] J. Elser et al., Appl. Phys. Lett. 89, 261102 (2006).

[16] A. L. Pokrovsky and A. L. Efros, Phys. Rev. B 65, 045110 (2002); M. Silveirinha, Phys. Rev. E 73, 046612 (2006); P. A. Belov, et al., Phys. Rev. B 67, 113103 (2003).

[17] J. Yao et al., Science 321, 930 (2008).

[18] M. Silveirinha, P. Belov, and C. Simovski, Phys. Rev. B 75, 035108 (2007); M. Silveirinha, P. Belov, and C. Simovski, Opt. Lett. 33, 1726 (2008); G. Shvets et al., Phys. Rev. Lett., 99, 053903 (2007).

[19] P. Evans et al., Nanotechnology 17, 5746 (2006).

[20] P. H. Lisseberger and R. G. Nelson, Thin Solid Films 21, 159 (1974).

[21] P. B. Johnson and R. W. Christy, Phys. Rev. B 6, 4370 (1972).

[22] Our commercial software (COMSOL, www.comsol.com) implements a model of periodic nanorod metamaterial with $\varepsilon_{\mathrm{Au}}$ given by Eq. (1), $\varepsilon_{\mathrm{Al}_{2} \mathrm{O}_{3}} \simeq 2.74$, and geometrical parameters $(r, d)$ deduced from the structures used in the experiments.

[23] The nonlocality appears on the macroscopic (metamaterial) scale; the response of each constituent of metamaterial has been considered to be local. 